\section{Fastfood für Gesunde}

\author{
C. Hurni
}

Gesundheitssprechstunden und Gesundheitsmagazine im Fernsehen erfreuen sich immer grösserer Beliebtheit. Unser Fernseharzt der Nation, Dr. Samuel Stutz, hat seine Fernsehpraxen voll und sowohl am Sonntagabend auf SF1 wie auch täglich auf Sat1 kann das Fernsehpublikum hautnah dem Praxisbesuch der Patientinnen und Patienten beiwohnen. Hautnah oft im wortwörtlichen Sinne, denn oft wird entblösste Haut auf der Untersuchungsliege gezeigt. Schliesslich

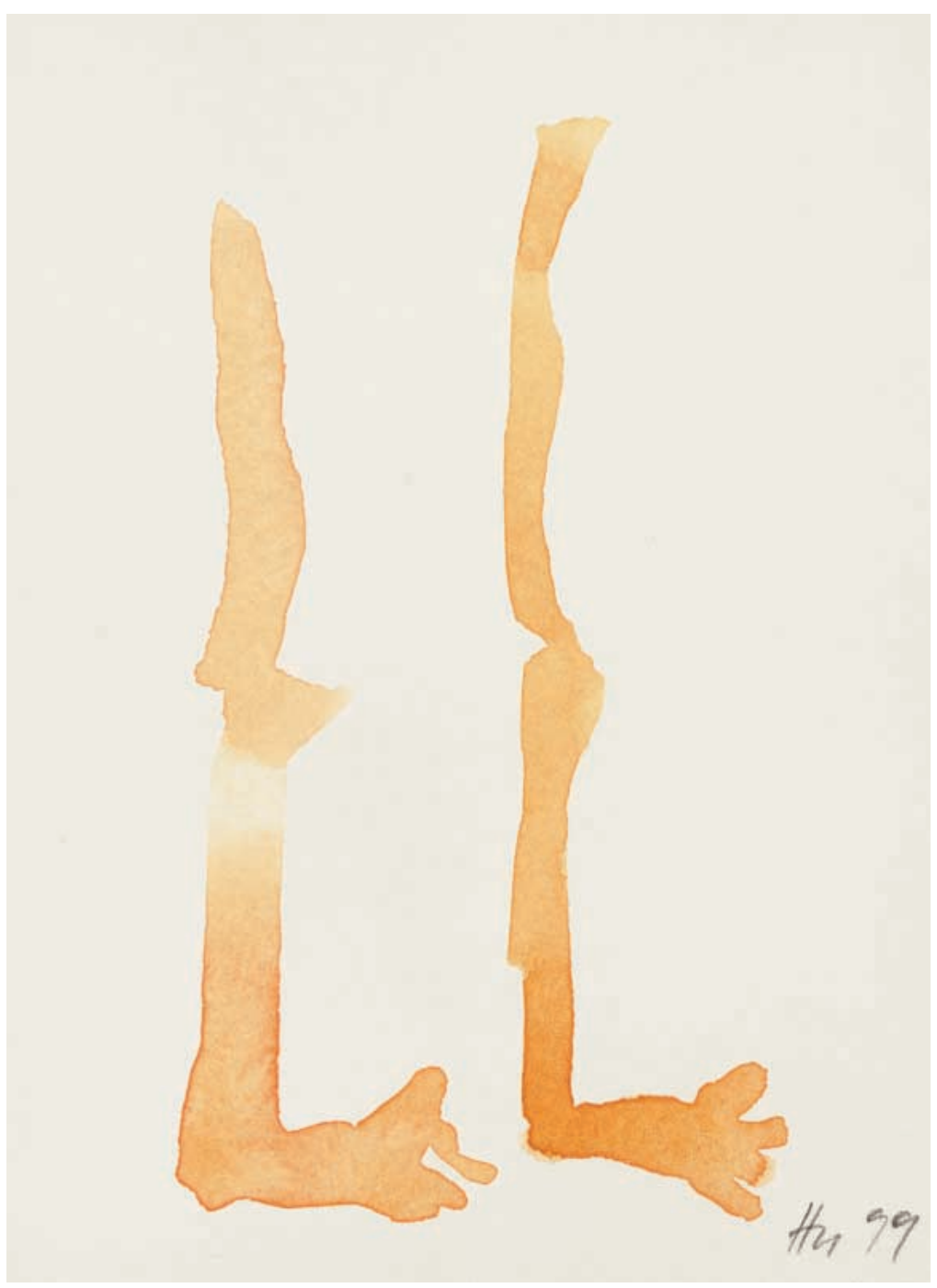

Korrespondenz:

Charlotte H. Hurni

Forchstrasse 251

CH-8032 Zürich

E-mail charlotte.hurni@)bluewin.ch soll das Drehbuch die Zuschauer bei der Stange halten und vermeiden, dass weitergezappt wird. Krankheit und Voyeurismus ist bestimmt eine prickelnde und brisante, aber in meinen Augen eine doch recht heikle Angelegenheit. Ich als kranke Person sehe mich jedenfalls nicht gerne im TV-Moulin-Rouge.

Dieses Regiekonzept ist vergleichbar mit einem Kochrezept. Man nehme eine Prise Krankheit, zeige Haut und lasse die Patientinnen und Patienten aus ihrem Erfahrungs- und Schicksalsrepertoire erzählen. Dr. med. Samuel Stutz garantiert als Chefkoch (mehr oder weniger) für das Gelingen der Gerichte. Er ist sozusagen der Dr. Oetker oder vielleicht besser die "Betty Bossy" der medialen Gesundheitsküche. Wie die Einschaltquoten zeigen, schmecken die servierten Speisen dem Publikum. Allerdings kann einem beim näheren Hinsehen der Appetit auch ein bisschen vergehen. Da werden manchmal kranke Leute regelrecht in die "Medien-Pfanne" gehauen und mit soviel Würze versehen, dass selbst diejenigen hinschauen, die im Grunde Angst vor Krankheit haben und das sind eigentlich alle. Krankheit, ein angstbesetztes Thema mit Unterhaltungswert, kann in diesen Sendungen in relativ kleinen Häppchen (homöopathisch quasi) und mit genügend Distanz "konsumiert» werden. Über allfällige Verdauungsschwierigkeiten der Zuschauerinnen und Zuschauer dieses Fastfoods oder Bauch- und Kopfschmerzen der "auftretenden Akteurinnen und Akteure" hat Dr. Stutz noch nie berichtet. Diese Beschwerden wären aber möglicherweise ebenfalls einmal eine Sendung wert?

Kürzlich bin ich als MS-Patientin ins Gesundheitsmagazin auf Sat1 eingeladen worden und "durfte" der Sendung die nötige Prise Krankheit beisteuern. Das Gericht ist zwar nicht zu meiner ganzen Zufriedenheit geraten, doch ist es den Umständen und Rahmenbedingungen entsprechend geniessbar herausgekommen. Die Erfahrung als Kranke auf der «Gesundheitsbühne" hat sich - nebst etwas Bauchweh und Lampenfieber - gelohnt und hinter die Kulissen $\mathrm{zu}$ schauen, finde ich eigentlich immer interessant. Doch jetzt der Reihe nach:

Das Rezept wurde bei mir etwas verfeinert, indem zusätzlich zum Studioauftritt ein paar Aufnahmen aus meinem Alltag gemacht wurden, die ich im Studio dann kommentierte. So begann für mich die Sendung bereits zwei Tage früher, unterwegs mit der Redaktorin und dem Kameramann im nahen Tobel, wo ich sehr häufig spazierengehe, in der Literarischen Agentur, wo ich zuletzt als Agentin gearbeitet habe, und beim Physiotherapeuten. Also ein bisschen "Leben heute" und "Leben vorher" der Charlotte H. Diese kleine Kamerareise durch einige Stationen aus meinem Leben mit MS waren physisch recht anstrengend für mich. Ganze drei Stunden waren wir zusammen unterwegs. Zum Glück waren meine "Reisebegleiter» äusserst angenehm und einfühlsam. Ich fühlte mich nie zu etwas gedrängt, was mir nicht behagt hätte, und wenn eine Pause fällig war, wurde sie - trotz Zeitdruck - selbstverständlich eingebaut. Dafür bin ich den beiden dankbar. 
Dann im Studio. Dr. Stutz' Praxis bei Sat1 ist funktional, kühl und steril eingerichtet. Die orangefarbenen Sessel, auf welchen er sich 1x täglich mit einer Fachärztin oder einem Facharzt und einer $\mathrm{Pa}$ tientin oder einem Patienten unterhält, sind fröhlich und sollen wohl ein «aufgestelltes Ambiente» vermitteln. Die vielen Leute, die da im Studio geschäftig und konzentriert herumschwirrten, beeindruckten mich, und die zahlreichen Gerätschaften, die an der Decke hängen oder herumstehen, sind faszinierend. Es waren noch andere Gäste geladen und mir schien, dass alle reichlich nervös waren. Da war es zeitweise gar nicht so einfach, sich nicht anstecken zu lassen und einen kühlen Kopf zu bewahren, und das Schminken bei der Stylistin kam gerade richtig, um sich einen Moment zurückzulehnen und zu entspannen. Auch das Resultat war sehr erfreulich. In der ersten Stunde wurde die Sendung rudimentär durchgeprobt und da und dort eine kleine Regieänderung vorgenommen. Dann galt es ernst. Kamera läuft und ab diesem Zeitpunkt wurde nichts - aber auch gar nichts mehr geändert. Also wach und bei der Sache sein! Zeitweise schwindelte mir bei der Vorstellung, auf dem orangenen Sofa zu sitzen, und ich fühlte mich wie damals in der Schule vor Prüfungen. Ich hatte Angst, mich plötzlich an überhaupt nichts mehr zu erinnern. Mein Kopf war stumpf und leer. Da ich mit den Aufnahmen zuletzt an der Reihe war und hinten im "Wartezimmer» sitzen durfte, nutzte ich die Gelegenheit, mir ununterbrochen aufzuzählen, was ich am Schluss gesagt haben wollte. Ich wusste, dass eine knappe Viertelstunde Sprechen spärlich sein würde, und das hat mich regelrecht gestresst.

Ich wurde dann also von der Praxishilfe gerufen, zog wie vorgesehen - aber dennoch ungern - meine Hose aus und legte mich auf die Untersuchungsliege. Die Kameras wurden auf mich gerichtet und der Neurologe war, als er kam, so hatte ich wenigstens den Eindruck, ziemlich durcheinander und hat an Stelle der vorgesehenen Reflex-Untersuchungen wie Babinski usw. vor allem meine Augenreflexe getestet. Viel Sinn hat die "Übung" nicht gemacht! Ich versuchte mich nicht aufzuregen und konzentrierte mich auf das folgende Gespräch, das dann mehr oder weniger zufriedenstellend ablief. Die beiden Ärzte waren, glaube ich, recht beeindruckt von meinen Schilderungen und Aussagen, jedenfalls haben sie selber nicht mehr viel gesagt oder gefragt. So kam mein "Statement" für das Publikum und vor allem für andere MS-Betroffene ein bisschen zusammen"gestutzt" und zu vereinfacht heraus, nämlich in etwa "Interferon spritzen und eine gute Einstellung finden, dann steht man wieder auf den eigenen Beinen ..." Das dürfte für viele, die das Medikament auch spritzen und gut mit ihrer Krankheit umgehen, aber den- noch keinen besseren Krankheitsverlauf erleben, sehr frustrierend sein. Oder für diejenigen mit «chronisch progredientem Verlauf", die gar kein Medikament zur Verfügung haben, erst recht. Überhaupt wurde der sehr unterschiedliche Verlauf der Krankheit und dessen mögliche Folgen in keiner Art und Weise erwähnt. Doch was kann in zwölf Minuten schon gesagt werden? Das ist wenig Zeit, sehr wenig. Wenn ich die Gelegenheit hätte, würde ich mindestens gerne ergänzen, dass ich offenbar auch Glück im Unglück habe, dass die Krankheit bei mir nach hartem Einstieg derzeit relativ mild verläuft.

Smalltalk kann ich die Art und Weise der geführten Konversation zwar nicht nennen, doch meiner Meinung nach ist, was dem Publikum da in der Regel im Gesundheitsmagazin aufgetischt wird, eine aufbereitete Kantinenmahlzeit, wenig nahrhaft, eher lieblos zubereitet und in aller Eile verschlungen. Die Sendungen sind Appetithäppchen à la "nouvelle cuisine» oder sogenannte "amuse-gueules», die hungrig machen, aber nicht satt. Für mein Gefühl konnte ich mich zwar als gute Vorspeise präsentieren, doch auf die Hauptspeise musste aus Zeitgründen leider verzichtet werden. Mein Anliegen war es, zu informieren und Sprachrohr für uns MS-Betroffene zu sein. Eine dermassen komplexe Krankheit wie MS kann in einer Viertelstunde unmöglich abgehandelt werden, und ich als einzelne kann auf keinen Fall alle Betroffenen vertreten. Da hatte ich zuviel erwartet, das sehe ich ein. Ich muss die Sendung so stehenlassen, wie sie ausgestrahlt wurde und mich mit dem Wissen zufriedengeben, dass ich mein Möglichstes getan und gegeben habe.

Ich will nicht ungerecht sein, aber kritisch. Das Fernsehteam war sehr nett zu mir, respektvoll und einfühlsam. Der Rahmen der Sendung müsste aber grundsätzlich überdacht werden. Vielleicht nicht aus fernsehtechnischer, jedoch aus ethischer Sicht. So mit Krankheit umzugehen, finde ich fragwürdig. Es wäre vielleicht sinnvoll und angebracht, solche Krankheiten in Dosierungen von meinetwegen einer Viertelstunde $\mathrm{zu}$ thematisieren, jedoch in fortgesetzter Folge, das heisst, beispielsweise eine Woche lang jeden Tag eine Sendung zur selben Krankheit mit allenfalls verschiedenen Betroffenen. So könnte vermutlich vertiefter und differenzierter informiert werden, ohne dass das Publikum wegschauen würde, also dennoch "fernsehtauglich». Ernsthafte Leiden so oberflächlich aufzugreifen und darzustellen, finde ich gefährlich. Das kann verunsichern, denn man weiss und weiss doch nichts. Mir persönlich behagt das nicht.

Ich plädiere an dieser Stelle für eine vollwertigere Mediengesundheitsküche und für "Mehrgänger», die satt machen im Sinne von wirklich informieren. 